\title{
Status of the commissioning of ATLAS
}

\author{
Sandro Palestini \\ CERN, CH 1211 Geneva 23 \\ on behalf of the ATLAS Collaboration
}

\begin{abstract}
The status of commissioning and readiness of the ATLAS experiment is reviewed, covering the installation of the last components, the test of the magnetic system, the combined runs with cosmic rays, the test of the computing system and the preparation for physics studies. The status of the detector with the first LHC beams in September 2008 is presented.
\end{abstract}

\section{INTRODUCTION}

After the phases of assembly and integration of the detector, ATLAS [1] is completing the commissioning work necessary for exploiting at best the start-up of LHC. The following sections will cover the progress made in the current year, and the status of the detector during the first circulation of beams, in September 2008.

\section{LAST PHASES OF INSTALLATION AND INTEGRATION}

Fig. 1 shows the general layout of ATLAS. A detailed description of all the components and 8 the expected performance has been published recently [2]. The last major steps in the integration of the detector are discussed in the following sections.

\subsection{End-cap stations of the muon spec-} trometer

The inner stations of the muon end-cap (Small Wheels) were installed in March and April 2008 (Fig. 2). They include Monitored Drift Chambers (MDT) and Cathode Strip Chambers (CSC) for precision tracking and measurement of muon momenta, and Thin Gap Chambers (TGC) for second coordinate and improved pattern recognition. Each wheel is also equipped with components of the alignment system. They measure the deformations and the positions of the precision chambers with respect to each other and to the chambers in the middle (Big Wheels) and outer (EO) stations.
The EO stations were completed in June, with MDT chambers and alignment bars mounted on the end walls of the ATLAS cavern. This operation was followed by the assembly of the last sections of the ATLAS beam pipe.

\subsection{Test of the magnetic system}

The magnetic system of ATLAS is formed by the solenoid (surrounding the inner tracking system, $r=1.3 \mathrm{~m},|z|<2.9 \mathrm{~m}$ ), the barrel toroid ( 8 coils in the region $5<r<10 \mathrm{~m}$ and $|z|<13 \mathrm{~m}$ ), and the end-cap toroids ( 8 coils in a single cryostat in the region $8<|z|<13 \mathrm{~m}$ on each side). While separate tests had been performed repeatedly in past years, combined tests of all components have started in July, and the full system has been operated since late August. Commissioning of the magnets included standard ramps to/from $20.5 \mathrm{kA}$ (7.6 kA in the solenoid) completed in few hours, and tests of emergency operations, such as fast dumps performed in three minutes, in which the cold-masses are heated to $51-59 \mathrm{~K}$.

\subsection{Commissioning of the inner detector}

Intense work took place on the ATLAS Inner Detector (ID) system in 2008, namely on the innermost component, the Pixel detector [3]. Cabling and connections were completed in spring, while final commissioning started in summer, after overcoming difficulties with the cooling system. Data taking periods with other subsystems have started in September. Fig. 3 shows one of the first cosmic rays detected in the Pixel and in the silicon micro-strip detector (SCT). As of late September, $94 \%$ of the Pixel modules are oper- 


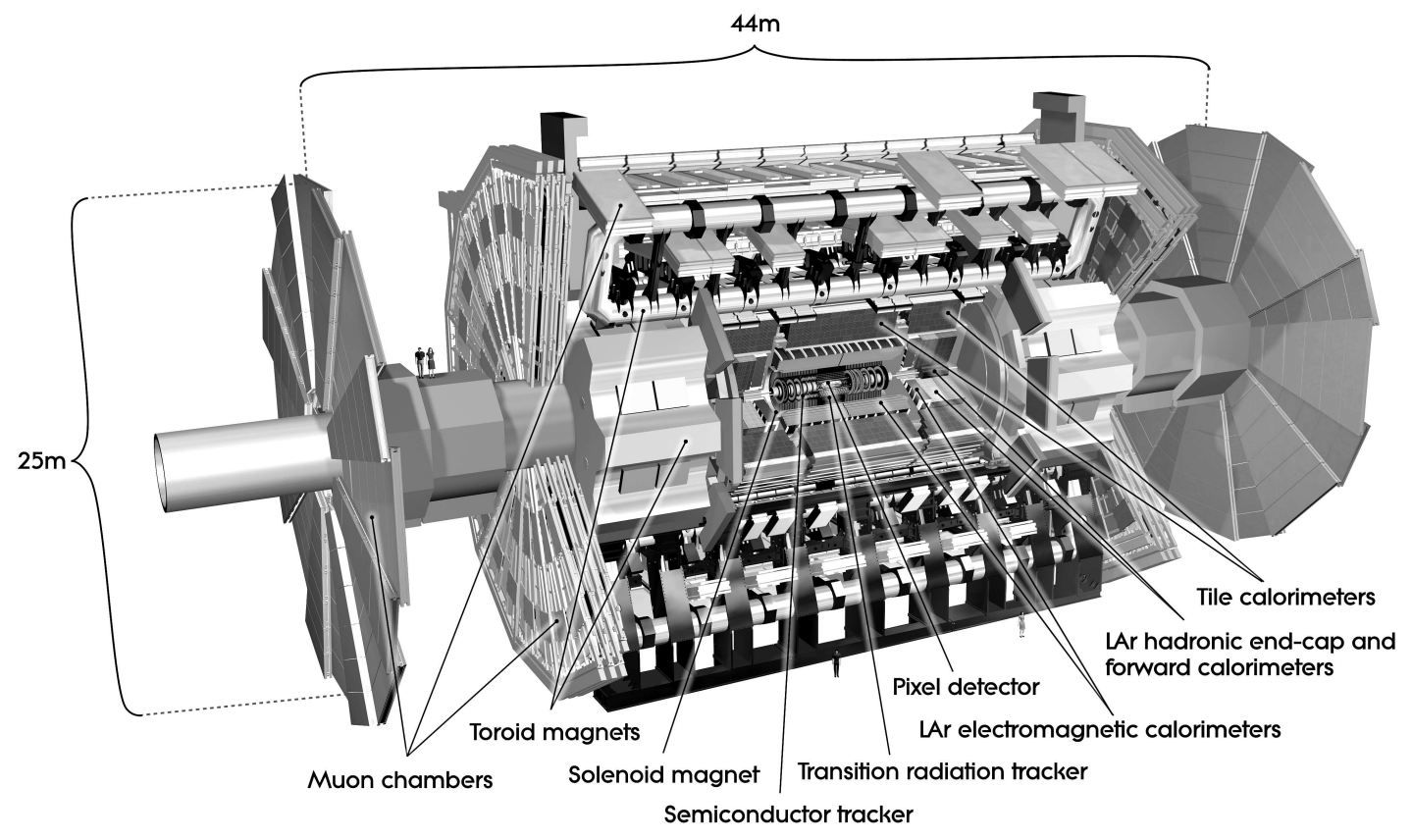

Figure 1. View of the ATLAS detector.

ational. The transition radiation tracker (TRT) completes the ATLAS ID. SCT and TRT have reached earlier an advanced level of commissioning $[4-6]$.

\subsection{Data Acquisition}

The Trigger-DAQ system is integrated and ready for commissioning runs and early physics [7]. The Level-1 trigger (based on the calorimeters and the muon system, and running at a maximum of $100 \mathrm{kHz}$ ) drives the transfer of event fragments from the sub-detectors to data buffers, and identifies regions-of-interest for which data is transferred to the Level-2 farm. The Level2 selection (with expected rate of $\sim 3 \mathrm{kHz}$ ) includes track reconstruction in the ID and drives the read-out of the full detector, and the eventbuilding. Level-2 also selects streams of data for calibration of the muon system. Final selections (to $\sim 100 \mathrm{~Hz}$ ) is performed in the event-filter farm, where full event reconstruction takes place, similarly to off-line data processing.

For 2008, special trigger menus have been pre- pared [8], with selection criteria suited to cosmicray events, beam-halo and low intensity collisions. The event size, which has a design value of 1.6 MB, is usually allowed a factor $2-8$ larger in commissioning runs, for a more detailed read-out of cells and samples in the e.m. calorimeter. In the period from August to October, an amount of $\simeq 1 \mathrm{~PB}$ of data has been recorded, comparable to the expectation for one year of LHC data.

Currently only of $35 \%$ of the CPUs are installed, with the aim of completing the system as the luminosity of LHC will increase in the coming years, hence optimizing cost and performance of the system.

\section{COSMIC RAYS RUNS}

Over the last two years, ATLAS has performed several combined runs with cosmic rays, which have been very helpful in the commissioning of the subsystems, in the integration of controls, trigger and data acquisition, and in the development and commissioning of reconstruction soft- 


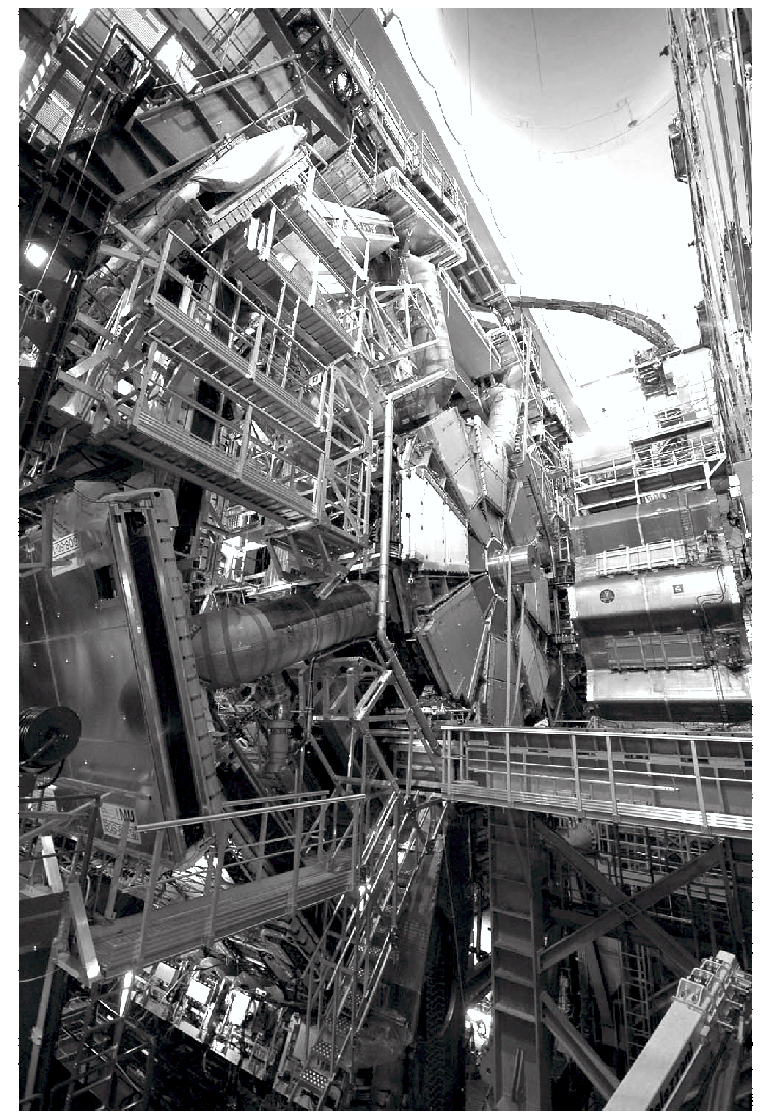

Figure 2. A Small Wheel of the muon system, during installation, near the center of the picture.

ware [9]. An event display for a cosmic event recorded in the muon system and in the TRT is shown in Fig. 4. The difference in measured angles between the two systems are at the level of $0.1-0.6 \mathrm{mrad}$, with r.m.s. width of about $10 \mathrm{mrad}$, in agreement with expectations for cosmic rays. The pattern recognition provided by the large numbers of drift-tubes layers in the TRT is proved in events with cosmic rays interactions near the ID, as shown in Fig. 5. Fig. 6 shows the energy deposition in clusters of the barrel e.m. calorimeter (a lead-liquid Argon sampling calorimeter with accordion-like cell geometry [10]). Cosmic muons have been studied also in the hadronic Tilecalorimeter (iron-scintillator tiles read-out with

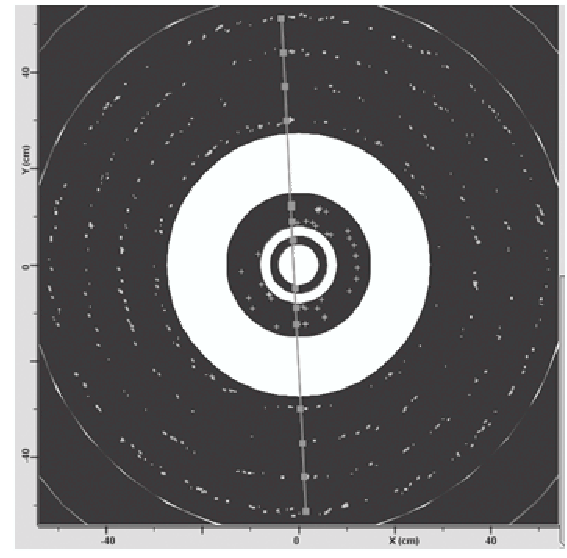

Figure 3. A cosmic ray crossing the beam pipe of ATLAS and detected on all layers of the Pixel and SCT systems.

wave-length shifting fibers).

\section{COMPUTING SYSTEM}

The computing model of ATLAS has been tested in two special "runs" (the FDR weeks) in winter and spring 2008. Luminosity values of $10^{31}$ and $10^{32} \mathrm{~cm}^{-2} \mathrm{~s}^{-1}$ respectively were chosen for the two exercises. Simulated data [11] were made available at the online storage after the eventfilter, and transferred to the local off-line production node (Tier-0), to ten Tier-1 sites in Europe, America and Asia for WLCG Grid production, and to a number of Tier-2 sites for analysis. The data transfer reached the value of $900 \mathrm{MB} / \mathrm{s}$, exceeding the design value of $700 \mathrm{MB} / \mathrm{s}$. Calibration [12] and analysis operations were included in the exercise, together with express-stream production for assessment of data quality. The computing model of ATLAS foresees that expressstream processing and calibration/alignment processing are performed within approximately 18 hours from data taking. A first bulk data processing can then occur locally at Tier-0, and (later) at the Tier-1 nodes. The full chain has been tested in June, identifying the areas were improvements are urgent, and demonstrating the validity of the design. 


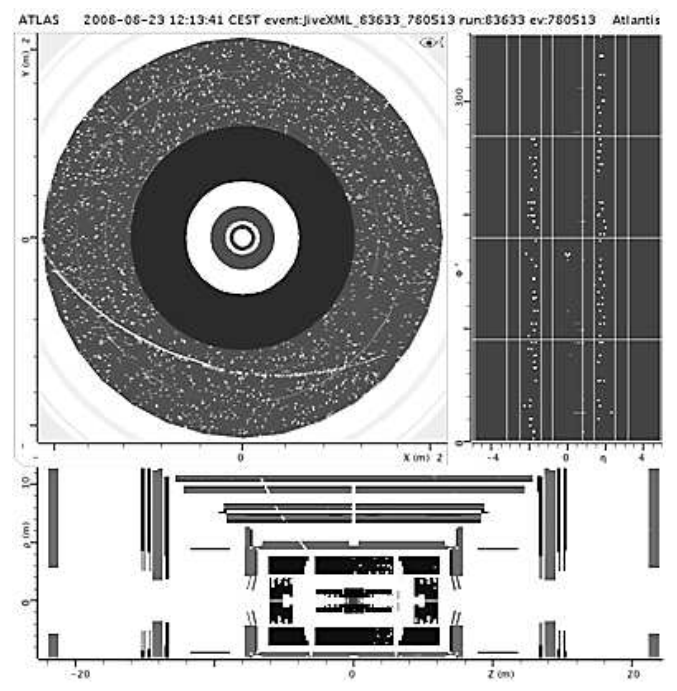

Figure 4. Event display of a cosmic muon crossing the muon and the TRT detectors.

\section{PREPARATION FOR PHYSICS}

ATLAS has recently completed a new set of physics studies (the CSC notes)[13], based on upto-date detector description and tools for reconstruction and analysis. The subjects covered include the performance of trigger, ID, muon system, b-tagging, electron and photon measurement, jets and missing transverse energy, and physics studies in the areas of Standard Model, $b$ physics, top, Higgs, SUSY and exotics. The main focus is on physics searches based on integrated luminosities in the range of $\sim 10-100 \mathrm{pb}^{-1}$.

\section{DETECTOR STATUS WITH FIRST BEAMS CIRCULATING IN LHC}

The ATLAS detector was operational when the first LHC beams circulated in September 2008, although for safety considerations the Pixel detector was switched off, and some other subsystems (e.g.: the SCT barrel, the Resistive Plate Chambers (RPC) of the muon barrel trigger) were operated with reduced voltage. As required for early beam operations, the solenoid magnet was off, but the toroid systems were operational.

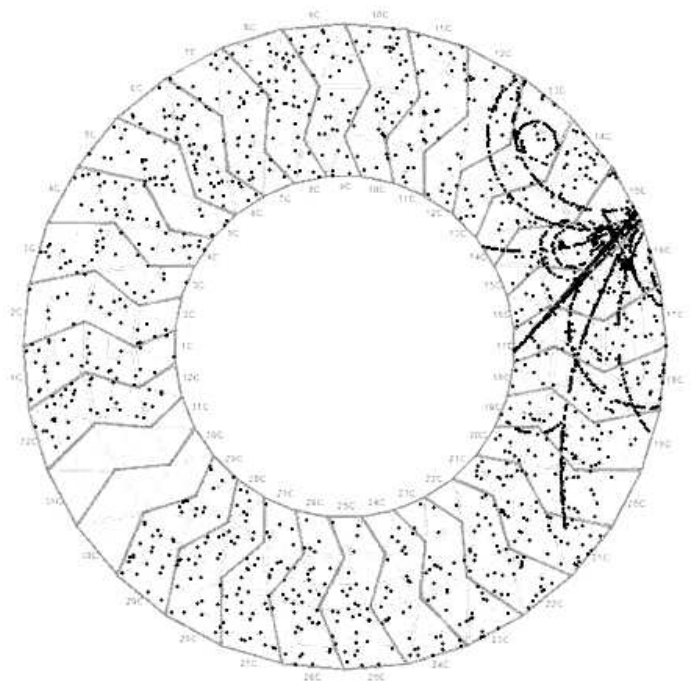

Figure 5. Reconstructed tracks in the TRT from a cosmic ray interacting inside ATLAS.

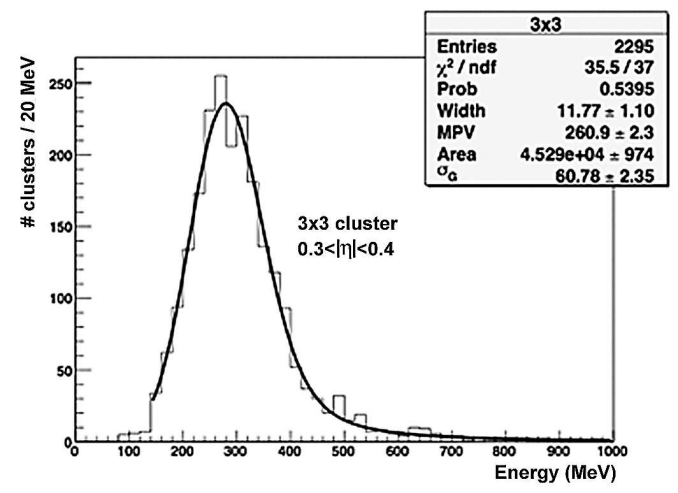

Figure 6. Muon energy spectrum in the e.m. barrel calorimeter.

The trigger [8] was based on Level-1 components with loose requirements on single tracks and energy clusters. They included a muon endcap trigger (TGC) and calorimetric triggers selecting clusters in the e.m. calorimeter (EM3), in the hadronic calorimeter (Tau5), and in the combination of the two (J5) (the numbers 3, 5 refer to nominal energy thresholds). In addition, 


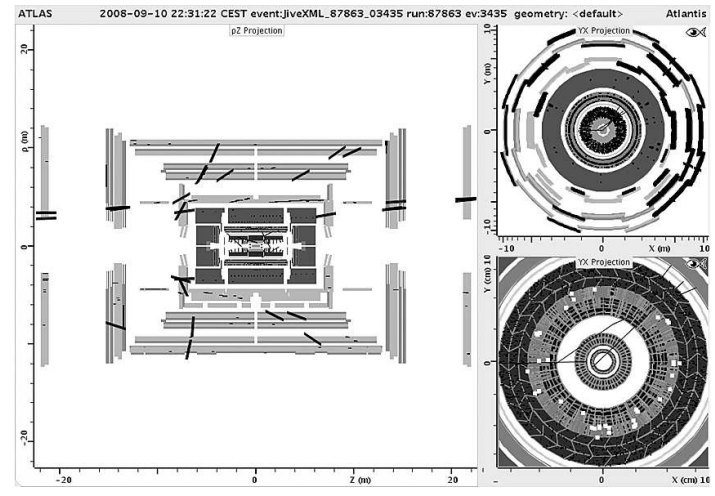

Figure 7. Event display for a beam-halo event. Muon tracks are bent away form the beam axis by the toroid magnets.

two tools have been set-up for early beams: a beam pick-up from the accelerator (BPTX), and a minimum-bias trigger (MBTS) derived from hodoscopes of plastic scintillators installed on the front face of the end-cap calorimeters.

The events recorded with the first beams circulating in LHC can be approximately classified as: (a) high-multiplicity events, when the beams were dumped on the tertiary collimators at $140 \mathrm{~m}$ from ATLAS, and (b) halo events, when the collimators were open and fewer muons crossed the detector. An event display from the latter class is shown in Fig. 7, and another, for the TRT detector, is shown in Fig. 8.

High multiplicity events are not suited for pattern recognition, but they provide useful checks. For example, detector timing can be tested. Fig. 9 shows the measured signal time in cells of the Tile-calorimeter, ordered according to the position along the beam axis: within each of the four sections of the detector, the dependence is dominated by the time-of-flight difference for particles moving along the beam axis, rather than originating in the center of ATLAS. Fig. 10 shows the relative time distribution for the different triggers, with the time-of-flight effect particularly visible for the muon trigger. Indeed, with the TGCBig Wheels located at $z= \pm 14 \mathrm{~m}$, the two trigger planes generate signals with time difference of

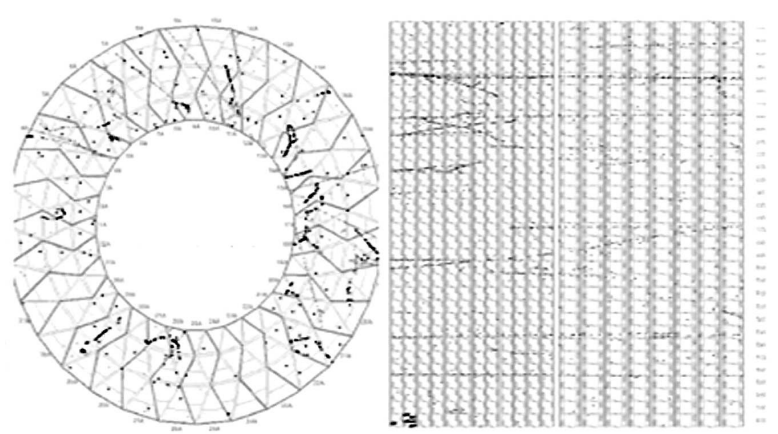

Figure 8. Beam-halo event in the TRT/Side A $(z>0)$ : tracks in barrel are projected on the plane $z=0$, tracks in the end-caps are projected on a cylindrical (unrolled) surface.

about $100 \mathrm{~ns}$, or four bunch crossing units.

\section{CONCLUSIONS}

After the impressive progress in installation and commissioning of most of the detector subsystems achieved in previous years, 2008 has seen a very significant progress in the overall commissioning of the detector, and in data handling, processing and analysis. Few hardware items remain to be integrated. Most online and offline procedures have been tested successfully. The exercise in September 2008 found us ready, and we are looking forward to collecting LHC data.

\section{REFERENCES}

1. Institutions member of the ATLAS Collaboration: Albany, Alberta, NIKHEF Amsterdam, Ankara, LAPP Annecy, Argonne NL, Arizona, UT Arlington, Athens, NTU Athens, Baku, IFAE Barcelona, Belgrade, Bergen, Berkeley LBL and UC, HU Berlin, Bern, Birmingham, UAN Bogota, Bologna, Bonn, Boston, Brandeis, Bratislava/SAS Kosice, Brookhaven NL, Buenos Aires, Bucharest, Cambridge, Carleton, Casablanca/Rabat, CERN, Chinese Cluster, Chicago, Chile, Clermont-Ferrand, Columbia, NBI Copen- 


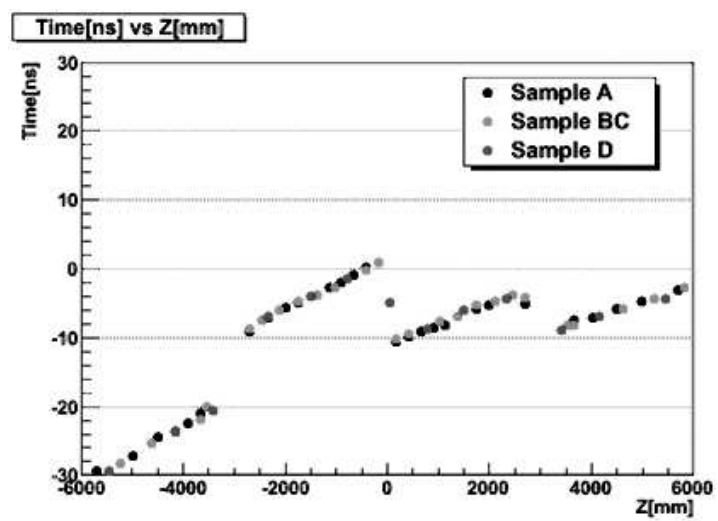

Figure 9. Timing in Tile-cal cells vs. longitudinal position: the slopes within each of the four sections are a time-of-flight effect of beam halo.

hagen, Cosenza, AGH UST Cracow, IFJ PAN Cracow, UT Dallas, DESY, Dortmund, TU Dresden, JINR Dubna, Duke, Frascati, Freiburg, Geneva, Genoa, Giessen, Glasgow, Göttingen, LPSC Grenoble, Technion Haifa, Hampton, Harvard, Heidelberg, Hiroshima, Hiroshima IT, Indiana, Innsbruck, Iowa SU, Irvine UC, Istanbul Bogazici, KEK, Kobe, Kyoto, Kyoto UE, Lancaster, UN La Plata, Lecce, Lisbon LIP, Liverpool, Ljubljana, QMW London, RHBNC London, UC London, Lund, UA Madrid, Mainz, Manchester, CPPM Marseille, Massachusetts, MIT, Melbourne, Michigan, Michigan SU, Milano, Minsk NAS, Minsk NCPHEP, Montreal, McGill Montreal, FIAN Moscow, ITEP Moscow, MEPhI Moscow, MSU Moscow, Munich LMU, MPI Munich, Nagasaki IAS, Nagoya, Naples, New Mexico, New York, Nijmegen, BINP Novosibirsk, Ohio SU, Okayama, Oklahoma, Oklahoma SU, Olomouc, Oregon, LAL Orsay, Osaka, Oslo, Oxford, Paris VI and VII, Pavia, Pennsylvania, Pisa, Pittsburgh, CAS Prague, CU Prague, TU Prague, IHEP Protvino, Regina, Ritsumeikan, UFRJ Rio de Janeiro, Rome I, Rome II, Rome III, Rutherford Appleton Laboratory, DAPNIA Saclay, Santa Cruz UC, Sheffield, Shinshu,

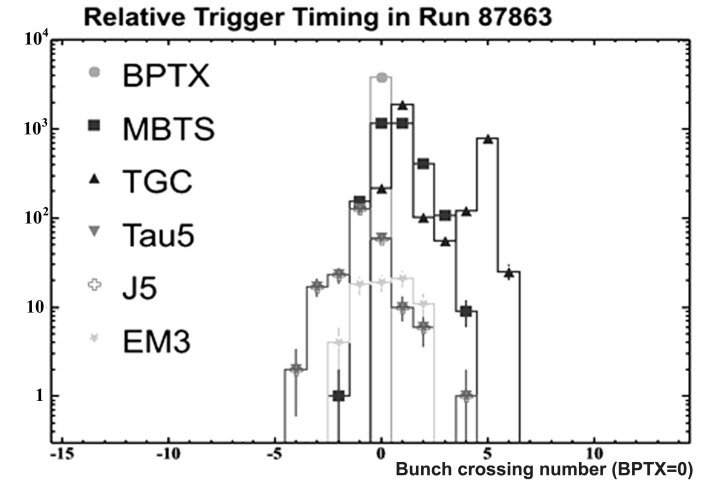

Figure 10. Relative timing of the different Level-1 triggers. Time-of-flight effects are most remarkable in the bimodal muon trigger (TGC) distribution.

Siegen, Simon Fraser Burnaby, SLAC, Southern Methodist Dallas, NPI Petersburg, Stockholm, KTH Stockholm, Stony Brook, Sydney, AS Taipei, Tbilisi, Tel Aviv, Thessaloniki, Tokyo ICEPP, Tokyo MU, Toronto, TRIUMF, Tsukuba, Tufts, Udine/ICTP, Uppsala, Urbana UI, Valencia, UBC Vancouver, Victoria, Washington, Weizmann Rehovot, FH Wiener Neustadt, Wisconsin, Wuppertal, Würzburg, Yale, Yerevan.

2. The ATLAS Collaboration, G. Aad et al., "The ATLAS Experiment at the CERN Large Hadron Collider", JINST 3 (2008) S08003.

3. See also N. Garelli, these proceedings.

4. See also J. E. Garcia, ibid.

5. See also P. Brückman de Renstrom, ibid.

6. See also A. Bocci, ibid.

7. See also W. Vandelli, ibid.

8. See also J. Masik, ibid.

9. See also Ch. Schmitt, ibid.

10. See also B. Trocmé, ibid.

11. See also Z. Marshall, ibid.

12. See also E. Solfaroli Camillocci, ibid.

13. The ATLAS Collaboration, "Expected Performance of the ATLAS Experiment, Detector, Trigger and Physics", CERN-OPEN2008-020, Geneva, 2008, to appear. 